\title{
Non-thermal atmospheric pressure plasmas as a novel candidate for preventive therapy of melanoma
}

\author{
Yasuhiro Omata $\cdot$ Machiko Iida $\cdot$ Ichiro Yajima • \\ Kozue Takeda $\cdot$ Nobutaka Ohgami $\cdot$ \\ Masaru Hori • Masashi Kato
}

Received: 25 June 2014 / Accepted: 3 July 2014/Published online: 19 July 2014

(C) The Japanese Society for Hygiene 2014

\begin{abstract}
Due to the increased ultraviolet radiation, the incidence of melanoma is increasing worldwide more than that of any other cancer. In this study, the effects of irradiation of non-thermal atmospheric pressure plasmas (NEAPPs) on benign melanocytic tumors from our original hairless model mice (HL-RET-mice), in which benign melanocytic tumors and melanomas spontaneously develop in the skin stepwise, were examined. Expression levels of melanoma cell adhesion molecule (MCAM) and matrix metalloproteinase-2 (MMP-2) mRNA in melanomas were higher than those in benign melanocytic tumors in the mice. Repeated irradiation of non-thermal atmospheric pressure plasmas (NEAPPs) for the benign tumors decreased the expression levels of MCAM and MMP-2 mRNA in the tumors from the mice. Previous studies
\end{abstract}

Electronic supplementary material The online version of this article (doi:10.1007/s12199-014-0399-1) contains supplementary material, which is available to authorized users.

Y. Omata $\cdot$ M. Iida $\cdot$ I. Yajima $\cdot$ N. Ohgami $\cdot$ M. Kato $(\square)$ Department of Occupational and Environmental Health, Nagoya University Graduate School of Medicine, 65 Tsurumai-cho,

Showa-ku, Nagoya, Aichi 466-8550, Japan

e-mail: katomasa@med.nagoya-u.ac.jp

Y. Omata $\cdot$ M. Iida $\cdot$ I. Yajima $\cdot$ K. Takeda $\cdot$ N. Ohgami

M. Kato

Department of Biomedical Sciences, College of Life and

Sciences, Chubu University, Kasugai, Aichi 487-8501, Japan

Y. Omata $\cdot$ M. Iida $\cdot$ N. Ohgami

Nutritional Health Science Research Center, Chubu University,

Kasugai, Aichi 487-8501, Japan

M. Hori

Department of Electrical Engineering and Computer Science, Graduate School of Engineering, Nagoya University, Furo-cho, Chikusa-ku, Nagoya 464-8603, Japan showed that MCAM sites are upstream of MMP-2, that MCAM regulates transcription of MMP-2 in melanoma cells and that MMP-2 is associated with the conversion of a benign tumor to a malignant tumor. Therefore, our results suggest that the NEAPP irradiation-mediated decrease in the expression level of MMP-2 in benign melanocytic tumors is associated with decreased expression levels of MCAM. Moreover, NEAPP irradiation might be a potential candidate for therapy to prevent melanoma development through suppression of malignant conversion in benign melanocytic tumors.

The incidence of melanoma, the most aggressive skin cancer, is increasing worldwide more than that of any other cancer [1]. However, effective therapies for preventing the development of melanoma are very limited. Thus, the establishment of effective therapies for melanoma is an important issue. We developed original hairless model mice carrying constitutively activated RET (HL-RETmice), in which benign melanocytic tumors and melanomas spontaneously develop in the skin stepwise $[1,2]$. The process of melanoma development in HL-RET-mice resembles the process of development of human large congenital melanocytic nevi, which are benign melanocytic tumors and might be a high risk factor for development of melanoma [1-3]. Previous studies showed that a mouse model might be a strong tool for the development of preventive therapies for melanoma $[1,2,4]$.

Plasma consists of an ionized gas and is defined as the fourth state of matter. Recent medical research has focused on the application of non-thermal atmospheric pressure plasmas (NEAPPs) for cancer therapy. Previous in vitro and in vivo studies showed anti-tumor effects of NEAPP irradiation on cancers [4-7]. However, there is limited 

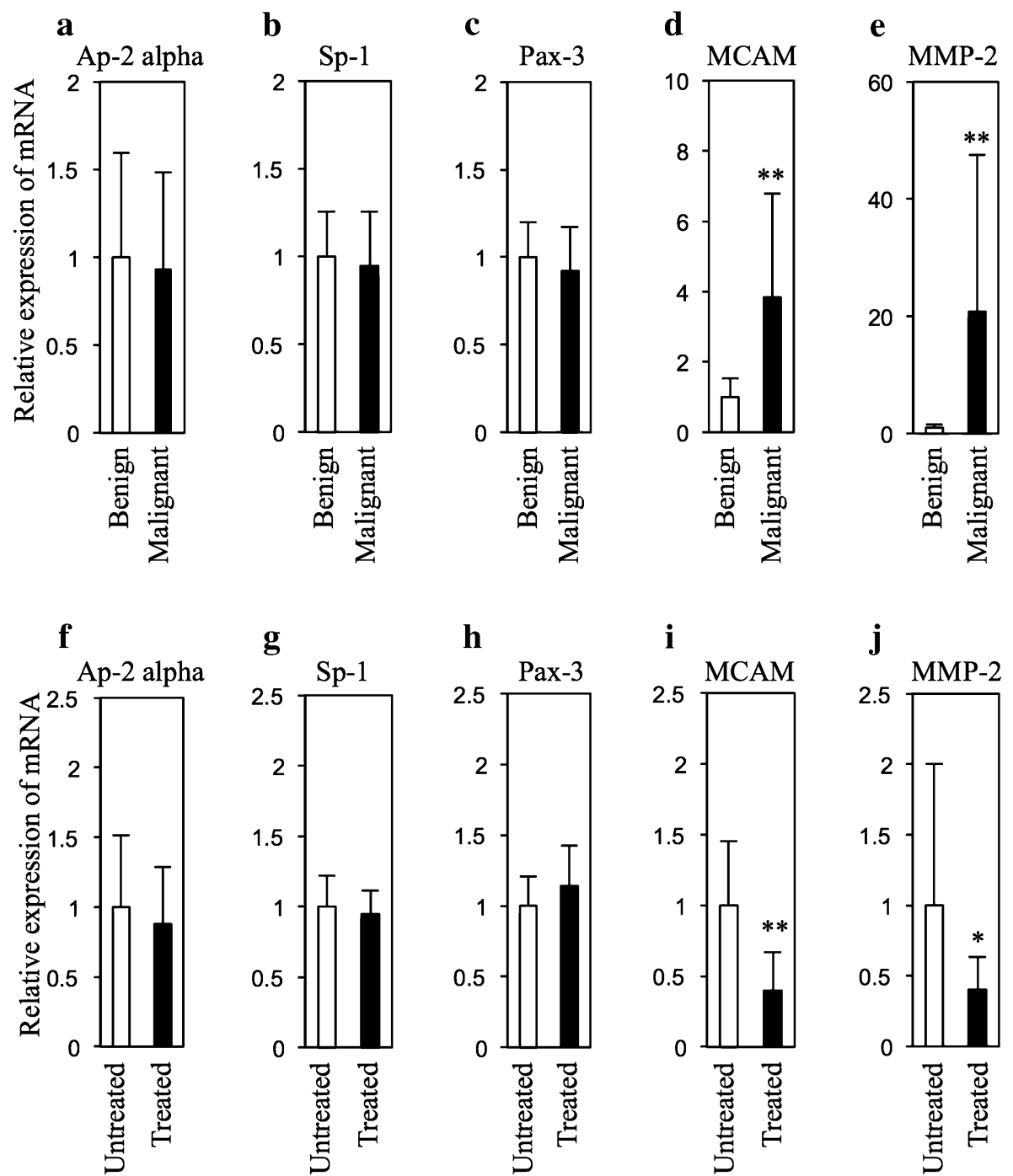

Fig. 1 Expression levels Ap-2 alpha, Sp-1, Pax-3, MCAM and MMP-2 in tumors from HL-RET-mice. Relative mRNA expression levels (mean $\pm \mathrm{SD}$ ) of Ap-2 alpha (a, f), Sp-1 (b, g), Pax-3 (c, h), $\operatorname{MCAM}(\mathbf{d}, \mathbf{i})$ and MMP-2 $(\mathbf{e}, \mathbf{j})$ in benign melanocytic tumors $(n=5)$ and melanomas $(n=5)(\mathbf{a}-\mathbf{e})$ and in NEAPP-treated $(n=5)$ and untreated $(n=5)$ benign melanocytic tumors $(\mathbf{f}-\mathbf{j})$ in HL-RET-mice are presented. The mRNA expression levels were measured by quantitative PCR and adjusted by the mRNA expression level of hypoxanthine ribosyltransferase (Hprt) according to the method previously described [4]. The method of NEAPP irradiation for

information on preventive effects of NEAPP irradiation on the process of malignant conversion from a benign tumor to a malignant tumor in vivo.

Our recent in vivo study showed that mRNA expression levels of matrix metalloproteinase-2 (MMP-2) in benign melanocytic tumors from HL-RET-mice were decreased by repeated NEAPP irradiation for 11 weeks after confirming that MMP-2 expression was increased in melanomas [4]. A previous study showed that melanoma cell adhesion molecule (MCAM) sites are upstream of MMP-2 and that benign melanocytic tumors in HL-RET-mice for 11 weeks in this study was previously described [4]. Sequences of primers used in analysis of quantitative PCR are presented in Supplemental Table 1. Statistical significance was evaluated by Student's $t$ test $(* p<0.05$, $* * p<0.01)$. The experiments using recombinant DNA were approved by the Recombination DNA Advisory Committee of Chubu University (approval no. 12-04) and Nagoya University (approval no. 12-59, 13-59, 13-76). The experiments using animals were approved by the Animal Care and Use Committee of Chubu University (approval no. 2510052) and Nagoya University (approval no. 26317)

MCAM regulates transcription of MMP-2 via Id-1 in melanoma cells [8]. Previous studies also showed that Ap-2 alpha, Sp-1 and Pax-3 are upstream of MCAM and regulate transcription of MCAM $[9,10]$. Therefore, in this study we focused on MCAM as a regulator of MMP-2 expression and Ap-2 alpha, Sp-1 and Pax-3 as regulators of MCAM expression (Supplemental Figure 1).

Our quantitative PCR analysis showed high mRNA expression levels of MCAM and MMP-2, but not Ap-2 alpha, Sp-1 and Pax-3 in melanomas compared to those in 
benign tumors (Fig. 1a-e), suggesting that MCAM and MMP-2, but not Ap-2 alpha, Sp-1 and Pax-3 are associated with the process of malignant conversion of benign melanocytic tumors. The repeated NEAPP irradiation for 11 weeks for benign melanocytic tumors decreased mRNA expression levels of MCAM and MMP-2, but not Ap-2 alpha, Sp-1 and Pax-3 (Fig. 1f-j). Moreover, a significant correlation $(R=0.72, p<0.01)$ between mRNA expression levels of MCAM and MMP-2 in benign melanocytic tumors from HL-RET-mice was shown by Spearman's rank-correlation coefficient analysis. A previous study showed that MMP-2 is associated with the conversion of a benign tumor to a malignant tumor [11]. Our recent in vivo study showed that repeated NEAPP irradiation for 11 weeks inhibited growth of benign melanocytic tumors through control of mRNA expression levels of cell cycle regulatory molecules in HL-RET-mice [12]. Taken together, our results suggest that the NEAPP irradiation-mediated decrease in the expression level of MMP-2 in benign melanocytic tumors is associated with decreased expression levels of MCAM but not Ap-2 alpha, Sp-1 and Pax-3. NEAPP irradiation is a potential candidate for therapy to prevent melanoma development through suppression of malignant conversion in benign melanocytic tumors. However, the mechanism of NEAPP-mediated decrease of MCAM expression remains unknown. NEAPP irradiation may modify the binding affinity of Ap-2 alpha, Sp-1 and Pax-3 to the promoter region of MCAM. Alternatively, NEAPP irradiation may modulate MCAM expression level via molecules other than Ap-2 alpha, Sp-1 and Pax-3. Further study is needed to clarify the above possibility in the future.

Acknowledgments This work was supported in part by Grants-inAid for Scientific Research (B) (No. 24390157 and 24406002), Grantin-Aid for Challenging Exploratory Research (No. 23650241 and 2667052), Grant-in-Aid for Scientific Research on Innovative Areas (No. 24108001), COE projects for Private Universities (Nutritional Health Science Research Center, No. 1201007) from the Ministry of Education, Culture, Sports, Science and Technology (MEXT); the Cosmetology Research Foundation; Toyoaki Scholarship Foundation; the Mitsui \& Co., Ltd. Environment Fund; Aichi Health Promotion Foundation and Research foundation from Center for Advanced Medicine and Clinical Research in Nagoya University Hospital.
Conflict of interest The authors have no conflict of interest to declare.

\section{References}

1. Kato M, Takahashi M, Akhand AA, Liu W, Dai Y, Shimizu S, et al. Transgenic mouse model for skin malignant melanoma. Oncogene. 1998;17:1885-8.

2. Thang ND, Yajima I, Nakagawa K, Tsuzuki T, Kumasaka MY, Ohgami N, et al. A novel hairless mouse model for malignant melanoma. J Dermatol Sci. 2012;65:207-12.

3. Marghoob AA. Congenital melanocytic nevi. Evaluation and management. Dermatol Clin. 2002;20:607-16.

4. Iida M, Yajima I, Ohgami N, Tamura H, Takeda K, Ichihara S, et al. The effects of non-thermal atmospheric pressure plasma irradiation on expression levels of matrix metalloproteinases in benign melanocytic tumors in RET-transgenic mice. Eur J Dermatol. 2014; in press.

5. Iseki S, Nakamura K, Hayashi M, Tanaka H, Kondo H, Kajiyama $\mathrm{H}$, et al. Selective killing of ovarian cancer cells through induction of apoptosis by nonequilibrium atmospheric pressure plasma. Appl Phys Lett. 2012;100:113702-4.

6. Tanaka H, Mizuno M, Ishikawa K, Nakamura K, Kajiyama H, Kano H, et al. Plasma-activated medium selectively kills glioblastoma brain tumor cells by downregulating a survival signaling molecule, AKT kinase. Plasma Med. 2013;3(3-4):265-77.

7. Utsumi F, Kajiyama H, Nakamura K, Tanaka H, Mizuno M, Ishikawa $\mathrm{K}$, et al. Effect of indirect nonequilibrium atmospheric pressure plasma on anti-proliferative activity against chronic chemo-resistant ovarian cancer cells in vitro and in vivo. PLoS One. 2013;8(12):e81576.

8. Zigler M, Villares GJ, Dobroff AS, Wang H, Huang L, Braeuer RR, et al. Expression of Id-1 is regulated by MCAM/MUC18: a missing link in melanoma progression. Cancer Res. 2011;71(10): 3494-504.

9. Mintz-Weber CS, Johnson JP. Identification of the elements regulating the expression of the cell adhesion molecule MCAM/ MUC18. Loss of AP-2 is not required for MCAM expression in melanoma cell lines. J Biol Chem. 2000;275(44):34672-80.

10. Medic S, Rizos H, Ziman M. Differential PAX3 functions in normal skin melanocytes and melanoma cells. Biochem Biophys Res Commun. 2011;411(4):832-7.

11. Overall CM, López-Otín C. Strategies for MMP inhibition in cancer: innovations for the post-trial era. Nat Rev Cancer. 2002;9:657-72.

12. Yajima I, Iida M, Kumasaka MY, Omata Y, Ohgami N, Chang J, et al. Non-equilibrium atmospheric pressure plasmas modulate cell cycle-related gene expressions in melanocytic tumors of RETtransgenic mice. Experimental Dermatol. 2014;23(6):424-5. 